\title{
Minimizing energy consumption in railways by voltage control on substations
}

\author{
A. U. Raghunathan ${ }^{1}$, T. Wada ${ }^{2}$, K. Ueda ${ }^{2} \&$ S. Takahashi ${ }^{2}$ \\ ${ }^{1}$ Mitsubishi Electric Research Laboratories, USA \\ ${ }^{2}$ Advanced Technology R\&D Center, Mitsubishi Electric Corporation, \\ Japan
}

\begin{abstract}
We propose an optimization method for minimizing energy consumption in DCelectrified railways where the trains are fitted with regenerative brakes. Reduction in energy consumption is achieved by controlling the voltage at the substations in real time. Conditional constraints are employed to model the behavior of trains and substations. This allows us to relax some of the imposed limits thereby enlarging the operating envelope of system and increasing the potential for energy savings. The conditional constraints are modeled using complementarity constraints which are smooth and differentiable. The optimization problem is an instance of a Mathematical Program with Equilibrium Constraints (MPEC). A numerical example with 42 trains and 8 substations is used to illustrate the savings from the method and the computational times. The proposed approach is shown to reduce energy consumption by about $3 \%$ over 2.5 minute of operation, recover about $97 \%$ of the regenerated energy while computing the optimal voltages in real time $(<1 \mathrm{~s})$.

Keywords: substation voltage control, regenerative braking, conditional models, complementarity constraints, mathematical programs with equilibrium constraints, energy reduction.
\end{abstract}

\section{Introduction}

Advances in traction motors, semiconductors and power electronic devices $[1,2]$ have all played a critical role in improving the energy efficiency of electrified railway systems worldwide. In particular, development of electrical regenerative braking has been a significant contributor in reducing the energy consumption. Decelerating trains produce electricity which is used to power accelerating 
trains by transmitting the regenerated power through the overhead lines. While significant effort has been invested in the development of device technology, there has been little research on the system coordination that has the potential to further increase the energy savings. In the DC power feeding system, the amount of power recovered from decelerating trains depends on catenary voltage at the decelerating train. On the other hand, utilization of such recovered power depends on the presence of accelerating trains with appropriate catenary voltage to receive it. In fact, the total regenerated power decreases with increasing voltage at the decelerating train. This goes counter to the conventional approach of operating networks at high voltages to limit losses in the network. If the power is not absorbed, catenary voltage rises, regenerative failure occurs, and kinetic energy is disposed with mechanical friction brake. This increase in voltage can damage the network lines. Consequently, it is necessary to determine the optimum voltage settings for the entire system that minimizes the energy obtained from substations connected to the electrical grid.

Miyatake and Ko [3] considered the problem of minimizing energy consumption while simultaneously controlling the train motion profile and substation voltages. The problem is formulated as an optimal control problem and solved using a steepest gradient approach. The approach was demonstrated on a 2 train system. The effect of timetable schedules was considered and shown to have significant impact on the savings. Miyatake and Ko [4] extended the problem to include on-board storage devices. However, the authors note that such a problem is difficult to solve in real-time $(<1 \mathrm{~s})$. Lu [5] considered heuristic rule-based power management strategies for railway systems. The problem of substation voltage control for real-time applications on a scale comparable to a typical real-world instance has not been considered. This is the focus of our work.

\subsection{Our contribution}

We consider the problem of determining the instantaneous voltages at the substations and the power drawn from decelerating trains so as to minimize the total power consumption from the substations while satisfying the power requirements for accelerating trains. It is assumed that at each sampling instant (typically $1 s$ ) the trains communicate to a central controlling station: (i) their locations, (ii) state of train - accelerating, coasting or decelerating and (iii) power demand if accelerating or maximum regenerated power if decelerating. The system architecture is shown in Figure 1. The motion profiles of the trains and timetables are pre-determined and are not included in the optimization problem. This can be viewed as the single time-instant version of the problem considered in [3]. However, in a departure from [3], we consider conditional modeling of substations that allows relaxing the operational limits when the substation does not provide power. This enlarges the operating envelope and provides for possibly increased energy savings. Conditional models for decelerating trains are also considered for a similar reason. Such models are inherently nonsmooth and hence, do not allow use of algorithms from smooth optimization. To alleviate this drawback we represent 


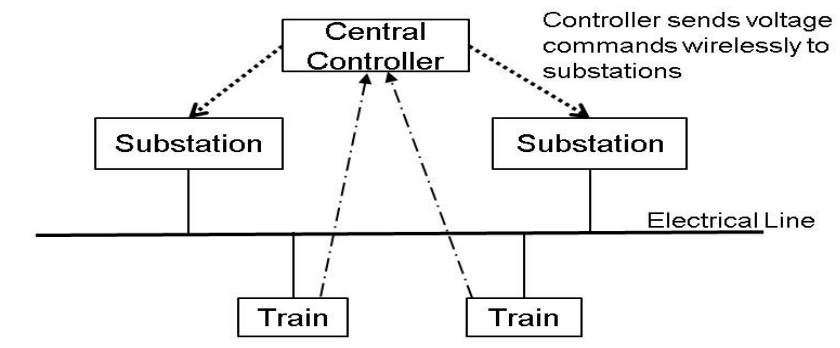

Trains wirelessly communicate: position, state and power requirement.

Figure 1: System architecture depicting the communication between central controller, substations and trains.

the conditional constraints using complementarity constraints. Consequently the optimization problem is an instance of a Mathematical Program with Equilibrium Constraints (MPEC) [6]. An extension of the algorithm in [7] is proposed which is computationally faster and more robust than the previous approaches. Results are provided on a system consisting of 8 substations and 42 trains.

\subsection{Organization of the paper}

The paper is organized as follows. Section 2 describes the mathematical model of the trains, substations and electrical network. Section 3 presents the optimization formulation and describes the algorithm. Results on a typical real-world instance is provided in Section 4, followed by conclusions in Section 5.

\section{Mathematical modeling of electrical network, substations and trains}

The electrical system associated with the electrical lines, substations, and trains is represented as a graph with nodes and edges. The set of all nodes in the graph is denoted by $\mathcal{N}$ while $\mathcal{N}^{S}, \mathcal{N}^{A}, \mathcal{N}^{R}$ denote respectively the set of substations, accelerating trains and regenerating trains. We assume without loss of generality that $\mathcal{N}^{S}, \mathcal{N}^{A}, \mathcal{N}^{R}$ are mutually disjoint and $\mathcal{N}^{S} \cup \mathcal{N}^{A} \cup \mathcal{N}^{R}=\mathcal{N}$. For convenience, coasting trains are included in the set $\mathcal{N}^{A}$ but do not demand any power. The edges in the graph are denoted by $\mathcal{L}$ and represent the electrical lines among the nodes in $\mathcal{N}$. We denote by $R_{i j}$ for $(i, j) \in \mathcal{L}$ the resistance on the electrical line connecting the nodes $i, j \in \mathcal{N}$. Observe that the electrical connections in the network are a function of train positions in relation to the fixed substations. The position of the trains changes with time. Consequently, $\mathcal{N}, \mathcal{L}$ are based on the instantaneous positions. 


\subsection{Substations without voltage control}

Substations $\left(i \in \mathcal{N}^{S}\right)$ are power sources for the railway network that receive alternating current (AC) from the grid and convert it to the direct current (DC) used by the trains. The set of substations whose voltages are not controlled is denoted by $\mathcal{N}^{S U} \subset \mathcal{N}^{S}$. The mathematical model for $i \in \mathcal{N}^{S U}$ is,

$$
I_{i} \geq 0, V_{i} \begin{cases}\geq V_{i}^{\max , S} & \text { if } I_{i}>0 \\ =V_{i}^{\max , S}-r_{i}^{S} I_{i} & \text { if } I_{i}=0\end{cases}
$$

where $V_{i}$ is the voltage at the node $i$ on the electrical line to which the substation is connected, $I_{i}$ denotes the current drawn from the substation, $V_{i}^{\max , S}$ is the maximum operational voltage at the substation and $r_{i}^{S}$ is the internal resistance of the substation. The non-negativity of $I_{i}$ prevents the substation from supplying power to the electrical grid. This restriction is not a limitation of our approach. Observe that the voltage at the substation can exceed $V_{i}^{\max , S}$ when no current is drawn. The voltage-current characteristic of substations $i \in \mathcal{N}^{S U}$ is shown in Figure 2(b).

\subsection{Voltage controlled substations}

The set of substations whose voltages are controlled is denoted as $\mathcal{N}^{S C} \subset \mathcal{N}^{S}$. The output voltage of substations can be controlled if they are equipped with Pulse Width Modulators (PWM). The mathematical model for such substations is,

$$
I_{i} \geq 0, V_{i} \in \begin{cases}{\left[V^{\min , S}, V_{i}^{\max , S}-r_{i}^{S} I_{i}\right]} & \text { if } I_{i}>0 \\ {\left[V_{i}^{\min , S}, \infty\right)} & \text { if } I_{i}=0\end{cases}
$$

where the $V_{i}^{\min , S}$ is minimum operational voltage at the substation parameters and variables above are identical to those in eqn (1). Observe that voltage at the feeding station has a discontinuity at $I_{i}=0$. In other words, eqn (2) imposes an upper limit on the voltage $V_{i}$ when current is drawn from the substation. If no current is drawn from the substations the substations $V_{i}$ is only limited by the minimum voltage. In Figure 3(a), the bold lines and the shaded area are the feasible values of voltages for given currents at voltage-controlled substations. Observe that when no current is drawn the entire voltage axis above $V^{\min , S}$ is feasible.

\subsection{Accelerating trains}

The accelerating trains $\left(i \in \mathcal{N}^{A}\right)$ represent the trains that are either accelerating or coasting and let $P_{i}^{A} \geq 0$ denote the power demanded by the trains. As explained earlier, it is assumed that the power demanded by each of these trains $P_{i}^{A}$ is given. The mathematical model is given by

$$
I_{i} \leq 0, V_{i} I_{i}=-P_{i}^{A}
$$




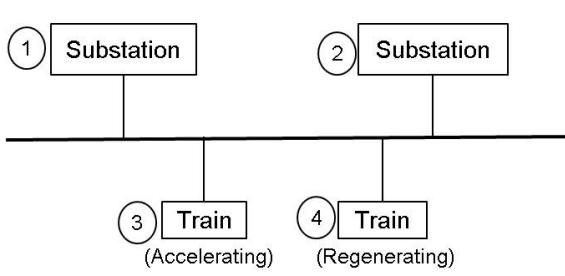

(a)

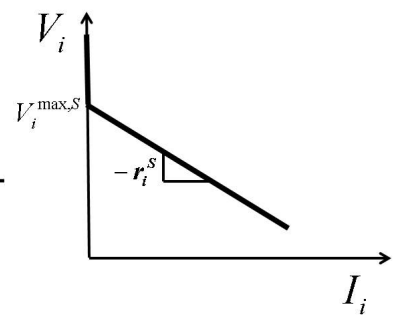

(b)

Figure 2: (a) Graph representation of the network. In this case, $\mathcal{N}=\{1,2,3,4\}$, and $\mathcal{L}=\{(1,3),(3,4),(4,2)\}$. Further, $\mathcal{N}^{S}=\{1,2\}, \mathcal{N}^{A}=\{3\}, \mathcal{N}^{R}$ $=\{4\}$. (b) Voltage-current characteristic of an uncontrolled substation.

where $V_{i}$ is the voltage at the node on the electrical line to which the accelerating train in connected, $I_{i}$ is the current drawn by the train and the non-positivity bound on current indicates that the current flows from the electrical lines to the trains. The voltage-current plot for power drawing trains is provided in Figure 3(b).

\subsection{Decelerating (regenerating) trains}

The decelerating trains $\left(i \in \mathcal{N}^{R}\right)$ can supply the power that is recovered from regenerative braking of the trains. However, the amount of power that is recovered from braking depends on the voltage at the electrical line and is modeled as:

$$
I_{i} \geq 0, V_{i}= \begin{cases}P_{i}^{R} / I_{i} & \text { if } V_{i} \leq V_{i}^{\min , R} \\ V_{i}^{\max , R}-\frac{V_{i}^{\max , R}-V_{i}^{\min , R}}{P_{i}^{R} / V_{i}^{\min , R}} I_{i} & \text { if } V_{i}^{\min , R}<V_{i} \leq V_{i}^{\max , R}\end{cases}
$$

In the above, $P_{i}^{R}$ is the maximum power that is available from the braking of the train and $V_{i}^{\min , R}, V_{i}^{\max , R}$ are some parameters associated with the regeneration device on the train. The regenerated power can be recovered in its entirety at voltages below $V_{i}^{\min , R}$. At higher voltages, the amount of power is curtailed to avoid damage to the regenerating device. This is done by imposing a decrease on the current $I_{i}$ that is proportional to the excess of the voltage over $V_{i}^{\min , R}$. For voltages higher than $V_{i}^{\max , R}$ no regenerated power is available from the decelerating train.

\subsection{Electrical network}

The circuit equations associated with the electrical circuit is:

$$
\left.\begin{array}{rl}
V_{i}-V_{j} & =R_{i j} I_{i j} \\
I_{i} & =-\sum_{j:(j, i) \in \mathcal{L}} I_{j i}+\sum_{j:(i, j) \in \mathcal{L}} I_{i j}
\end{array}\right\} \forall(i, j) \in \mathcal{L},
$$




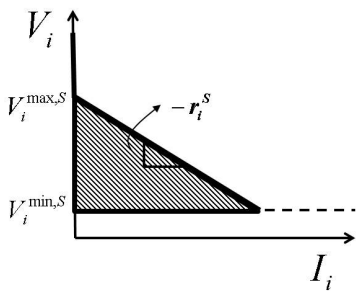

(a)

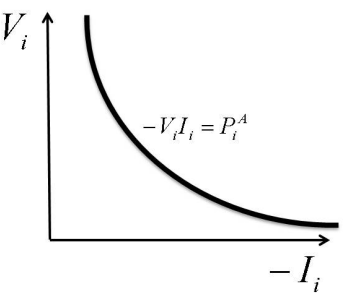

(b)

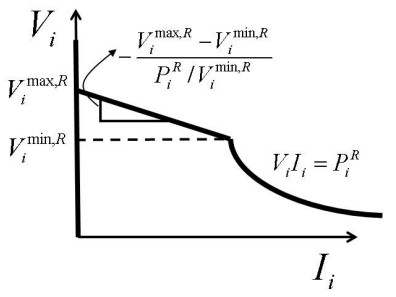

(c)

Figure 3: Voltage-current characteristics of (a) substations, (b) accelerating trains and (c) decelerating trains.

which are the Kirchoff's Laws. In the above, $V_{i}$ denotes the voltage on the electrical line at node $i, I_{i}$ is the current injected into the electrical line at node $i$, and $I_{i j}$ denotes the current on the line $(i, j) \in \mathcal{L}$. Note that we have used the convention that $I_{i j}>0$ when current flows from $i$ to $j$ for $(i, j) \in \mathcal{L}$.

\section{Mathematical Program with Complementarity Constraints (MPCC)}

The models for the substations eqns (1), (2) are non-differentiable. Most nonlinear programming algorithms assume differentiability of constraints and are inapplicable to eqns (2), (2). Another approach to address the non-differentiability is to choose a priori for each substation the differentiable piece on which the solution lies and then, solve a smooth nonlinear program. However, that will entail solving an exponentially large number $\left(\sim 2^{\left|\mathcal{N}^{S}\right|}\right)$ of nonlinear programs. More importantly, not all of these may be feasible. To obtain a tractable formulation. we propose to address the non-differentiability by modeling using complementarity constraints. This leads to other issues for optimization but these have been successfully addressed in the literature as explained in Section 3.4.

\subsection{Subststation without voltage control}

We propose the following exact reformulation of the model in eqn (2) using complementarity constraints. In particular, the model for $i \in \mathcal{N}^{S U}$ is,

$$
\begin{aligned}
& V_{i}=V_{i}^{\max , S}-r_{i}^{S} I_{i}+s_{i}, \\
& I_{i}, s_{i} \geq 0, I_{i} s_{i}=0 .
\end{aligned}
$$

In the above, we introduced a scalar $s_{i}$ to model the excess of the substation voltage over the maximum operational voltage $V_{i}^{\max , S}$ when $I_{i}=0$. In addition, $s_{i}$ is restricted to be nonnegative and also the product of $I_{i}$ and $s_{i}$ is required to be zero. The last constraint in eqn (6) is the so called complementarity constraint. It 
requires that at any feasible solution either $I_{i}$ or $s_{i}$ vanishes. Consequently, when $I_{i}>0$ the scalar $s_{i}=0, V_{i}=V^{\max , R}-r_{i}^{S} I_{i}$ is imposed. On the other hand when $I_{i}=0$, the scalar $s_{i} \geq 0$ can assume a positive value thereby allowing the voltage to exceed $V_{i}^{\max , S}$. More importantly, the above constraints are all differentiable.

\subsection{Voltage controlled substation}

In an analogous manner to eqn (6), we reformulate eqn (2) using complementarity constraints. In particular, the model for $i \in \mathcal{N}{ }^{S C}$ is,

$$
\begin{aligned}
& V_{i}^{\min , S} \leq V_{i} \leq V_{i}^{\max , S}-r_{i}^{S} I_{i}+s_{i}, \\
& I_{i}, s_{i} \geq 0, I_{i} s_{i}=0 .
\end{aligned}
$$

The equivalence of eqn (2) and eqn (7) can be shown using arguments similar to those in Section 3.1.

\subsection{Relaxed regenerating train}

The regenerating train model eqn (4) requires that voltage and current lie on two pieces - the curve $V_{i} I_{i}=P_{i}^{R}$ or the straight line. Clearly this model is nondifferentiable. We propose to relax the constraints in eqn (4) as follows,

$$
V_{i} \leq \begin{cases}P_{i}^{R} / I_{i} & \text { if } V_{i} \leq V_{i}^{\min , R} \\ V_{i}^{\max , R}-\frac{V_{i}^{\max , R}-V_{i}^{\min , R}}{P_{i}^{R} / V_{i}^{\min , R}} I_{i} & \text { if } V_{i}^{\min , R}<V_{i} \leq V_{i}^{\max , R} \quad, I_{i} \geq 0 .\end{cases}
$$

This relaxation amounts to allowing the area under the graph in Figure 3(c) to be feasible as opposed to just the boundary. Since the optimization problem minimizes the amount of power drawn from the substations we expect to utilize all of the regenerated energy for powering the accelerating trains at an optimal solution. We cannot prove this statement but show in Section 4 through numerical experiments that the solution obtained using eqn (8) does indeed satisfy eqn (4). Also, upper limit on the voltage is not imposed when no current is drawn from the decelerating train. This is modeled using complementarity constraints as:

$$
\begin{aligned}
& V_{i} I_{i} \leq P_{i}^{R}+s_{i}, \\
& V_{i} \leq V^{\max , R}-\frac{V_{i}^{\max , R}-V_{i}^{\min , R}}{P_{i}^{R} / V_{i}^{\min , R}} I_{i}+s_{i}, \\
& I_{i}, s_{i} \geq 0, I_{i} s_{i}=0 .
\end{aligned}
$$

\subsection{Optimization formulation}

We are interested in regulating the voltages at substations so that the power that is drawn from the substations is minimized. Utilizing the models described in the 
previous section the optimization problem can be formulated as:

$$
\begin{array}{ll}
\min & \sum_{i \in \mathcal{N}^{S}} V_{i} I_{i} \\
\text { s.t. } & \text { eqns }(3),(5),(6),(7),(9) .
\end{array}
$$

The optimization problem in eqn (10) is an instance of a Mathematical Program with Equilibrium Constraints (MPEC) [6]. MPECs are a class of nonlinear programs that include as constraints, complementarity constraints. MPECs have been used to model problems in economics, traffic [6], structural engineering [8], chemical engineering [9], bioengineering [10] among others.

MPECs possess a number of numerical deficiencies [6]. Importantly, the feasible region for MPECs do not have a strict interior. This precludes the use of interior point algorithms $[11,12]$ which have shown to be successful in solving largescale inequality constrained nonlinear programs. These algorithms require a strictly feasible interior for the constraints. However, several studies [7, 13] have reformulated the complementarity constraints, with a strictly feasible interior, so that interior point algorithms can be applied. In particular, we relax the complementarity constraints in eqn (10) as,

$$
I_{i} s_{i} \leq \eta_{i} \forall i \in \mathcal{N}^{S} \cup \mathcal{N}^{R}
$$

where $\eta_{i}>0$ is a chosen parameter. The above relaxation of complementarity constraint endows the optimization problem with an interior and makes it amenable to solution using interior point algorithms. Observe that as $\eta_{i} \rightarrow 0$ we recover the complementarity conditions. Motivated by this we solve a sequence of optimization problems for $\left\{\eta_{i}^{k}\right\} \forall i \in \mathcal{N}^{S} \cup \mathcal{N}^{R}, k=0,1,2, \ldots$ monotonically decreasing. We refer the interested reader to Raghunathan and Biegler [7] for more details.

\section{Numerical experiments}

In this section, we present numerical results on an electrical railroad covering about $32 \mathrm{~km}$ with 23 stations. We consider 2.5 minutes of train operation.

\subsection{Problem setup}

There are 8 substations along the railroad located at following distances in $\mathrm{km}$ : $1.07,5.55,8.45,12.29,16.66,22.01,25.54$ and 30.09. The electrical railroad has two lines running in opposite direction. Figures 4(a) and 4(b) show respectively the net power demanded by trains $\left(=\sum_{i \in \mathcal{N}{ }^{A}} P_{i}^{A}\right)$ and net power regenerated by braking trains $\left(=\sum_{i \in \mathcal{N}^{R}} P_{i}^{R}\right)$. Of course, the location of the power demand and power regeneration for different trains depends on the run-curve of the trains. Based on the assumed run-curves, the net energy demanded by the trains over the 2.5 minute period is $979.5 \mathrm{kWh}$. The net energy available from the braking of the trains is $497.2 \mathrm{kWh}$. The parameters for substations are set as: 


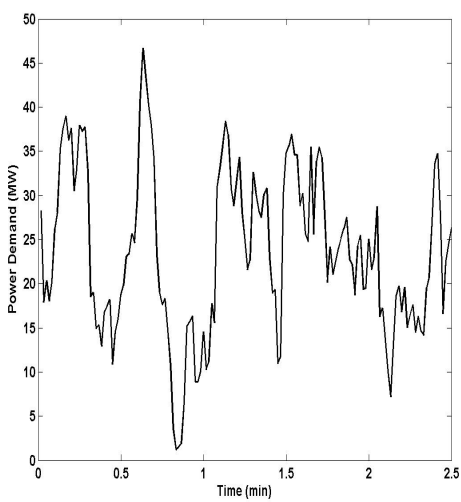

(a)

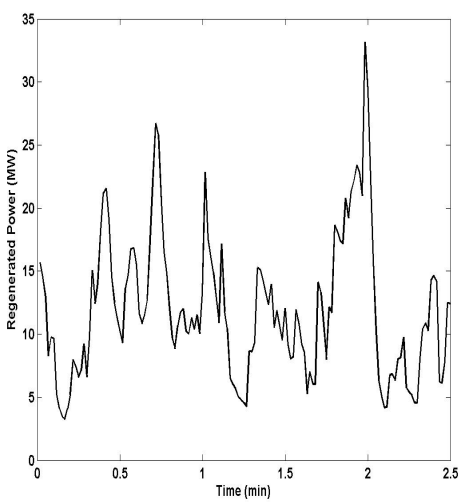

(b)

Figure 4: Time profiles of (a) Net power demand, (b) Net regenerated power.

$V_{i}^{\max , S} \in(1.58,1.68) \mathrm{kV}, V_{i}^{\min , S}=1.3 \mathrm{kV}$ and $r_{i}^{S} \in(0.011,0.29) \Omega(\mathrm{Ohm})$. For the regenerating trains, $V_{i}^{\max , R}=1.75 \mathrm{kV}$ and $V_{i}^{\min , R}=1.7 \mathrm{kV}$. The resistivity of lines is set to $42.7 \mathrm{~m} \Omega / \mathrm{km}$.

\subsection{Results}

We considered two cases of substation operation:

- No Voltage Control - In this scenario, $\left|\mathcal{N}^{S U}\right|=8, \mathcal{N}^{S C}=\emptyset$.

- Voltage Control - In this scenario, $\mathcal{N}^{S U}=\emptyset,\left|\mathcal{N}^{S C}\right|=8$.

The computations were performed on Intel i7-3930K $3.6 \mathrm{GHz}$ CPU with $16 \mathrm{~GB}$ RAM running Windows 7. The average CPU times for No Voltage Control was $0.44 \mathrm{sec}$ while for No Voltage Control was $0.66 \mathrm{sec}$. In the latter case, there were 11 instances where the CPU time was $>1 \mathrm{sec}$ with max CPU time of $1.78 \mathrm{sec}$.

Table 1 presents the comparison of results for the two cases. An inspection of results in Table 1 shows that controlling the substation voltages reduces the energy consumed from substations by $24.69 \mathrm{kWh}$. This represents an energy savings of $3 \%$ over the No Voltage Control scenario. More importantly, $97 \%$ of the regenerated energy from braking trains is recovered in the Voltage Control case. On the other hand, in the No Voltage Control scenario only $89.2 \%$ of regenerated energy is recovered. Figure 5 plots the net power supplied from all substations at different time instances for the two cases. The greatest difference between the two profiles occurs simulation time of $t=25 \mathrm{sec}$ (indicated by dotted vertical line in Figure 5). Figure 6 plots profile of the voltages along the length of the railroad at $t=25 \mathrm{sec}$. Observe that for Voltage Control the substations (denoted by $*$ ) and regenerating trains (denoted by $\circ$ ) operate at lower voltages. Thus, higher energy recovery is achieved in the Voltage Control as shown in Figure 7. Further, note that substations SS1, SS6, SS8 do not supply power (refer Figure 7) and in No 
Table 1: Comparison of results for No Voltage Control and Voltage Control cases.

\begin{tabular}{|l|c|c|}
\hline & No Voltage Control & Voltage Control \\
\hline Energy from substations $(\mathrm{kWh})$ & 814.9 & 790.2 \\
\hline Energy from braking trains $(\mathrm{kWh})$ & 443.4 & 482.1 \\
\hline Total energy $(\mathrm{kWh})$ & 1258.3 & 1272.3 \\
\hline$\%$ energy recovered from braking trains & 89.2 & 97.0 \\
\hline
\end{tabular}

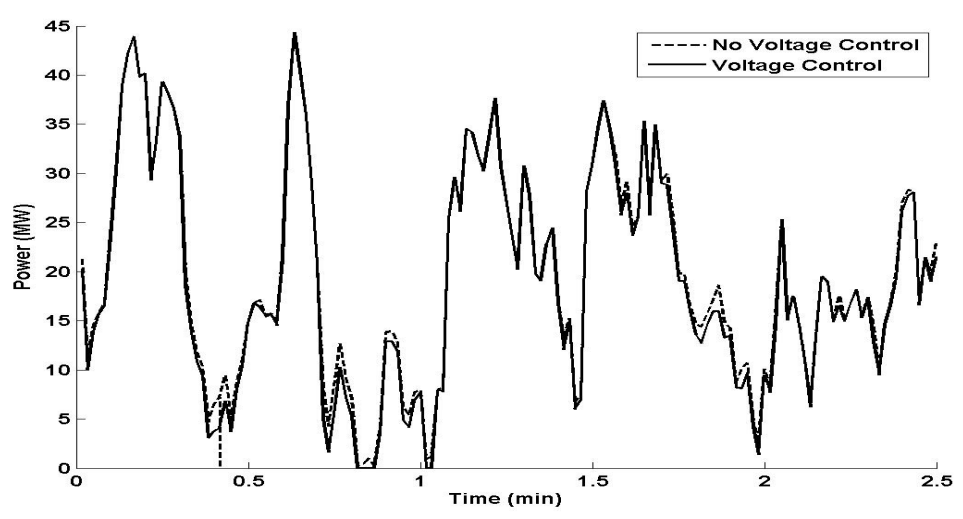

Figure 5: Optimal time profiles of power supply from substations for No Voltage Control and Voltage Control cases.

Voltage Control case the voltages at these substations exceed $V_{i}^{\max , S}$ (denoted by $\triangle$ in Figure 6). Trains A19,A18, B11, B12, A2 recover more regenerated energy in Voltage Control case. In our numerical experiments, we have observed that eqn (4) is satisfied for all time instants expect for $t=49 \mathrm{sec}$. At $t=49 \mathrm{sec}$, the net demand from accelerating trains is only $3.55 \mathrm{MW}$ while the regenerating trains produce as much as $9.79 \mathrm{MW}$. Hence, at the optimal solution the energy recover is greatly reduced to avoid exceeding the demand and this leads to a large error. This justifies the use of the relaxation in eqn (9).

The results clearly demonstrates, as outlined in the introduction, that the high catenary voltage reduces the recovered energy from braking trains. However, we do notice that the total amount of energy supplied in the Voltage Control case exceeds that of the No Voltage Control case. This can be explained through increased line losses in the former case wherein braking trains are possibly located further away from power demanding trains. In such scenarios, it may be beneficial to supply energy from substations that are closer to the power demanding trains and store the regenerated energy. We plan to explore this in a future work. 


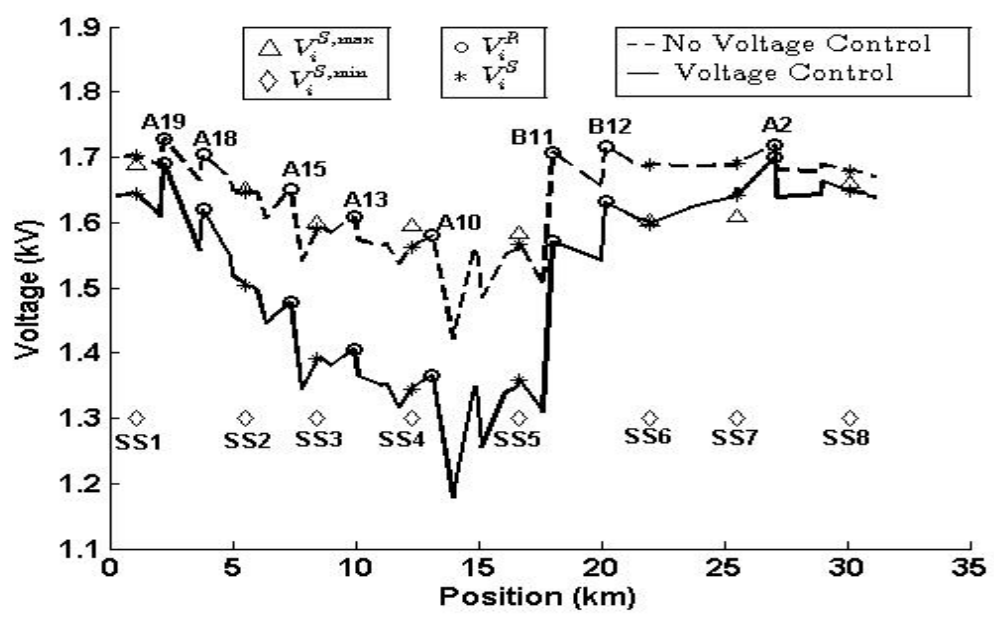

Figure 6: Optimal voltages along length of railroad at simulation time of $25 \mathrm{sec}$.

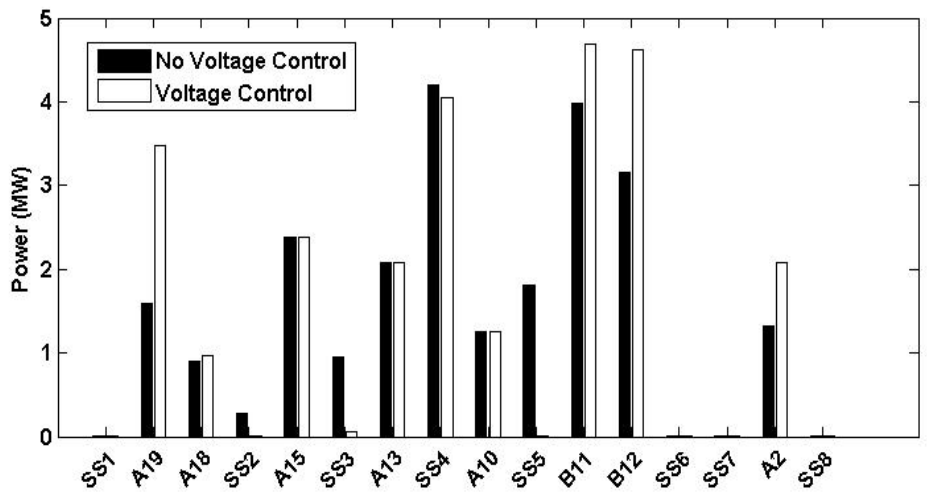

Figure 7: Power supplied by substations and regenerating trains at simulation time of $25 \mathrm{sec}$.

\section{Conclusions}

We proposed a novel approach for controlling the substation voltages so as to minimize the energy consumption in railway operations. Complementarity based modeling was presented as a computationally efficient approach for handling conditional models. The numerical results clearly demonstrate the role of substation voltage control in reducing the energy consumption by about $3 \%$ by increasing recovered energy from braking trains over 2.5 minutes of railroad 
operation. Further, the proposed solution approach enables real-time control as it is robust and computationally fast on standard desktop processors. The influence of storage devices on energy consumption will be considered in a future study.

\section{References}

[1] Steimel, A., Power-electronics issues of modern electric railway systems. Advances in Electrical and Computer Engineering, 10(2), pp. 3-10, 2010.

[2] Uzuka, T., Trends in high-speed railways and the implications on power electronics and power devices. 23rd International Symposium on Power Semiconductor Devices and IC's, IEEE, pp. 6-9, 2011.

[3] Miyatake, M. \& Ko, H., Numerical analyses of minimum energy operation of multiple trains under DC power feeding circuit. European Conference on Power Electronics and Applications.

[4] Miyatake, M. \& Ko, H., Optimization of Train Speed Profile for Minimum Energy Consumption. IEEJ Transactions on Electrical and Electronics Engineering, 5(3), pp. 263-269, 2010.

[5] Lu, S., Optimising Power Management Strategies for Railway Traction Systems. Ph.D. thesis, 2011.

[6] Luo, Z.Q., Pang, J.S. \& Ralph, D., Mathematical Programs with Equilibrium Constraints. Cambridge University Press, 1996.

[7] Raghunathan, A.U. \& Biegler, L.T., An interior point method for mathematical programs with complementarity constraints (MPCCs). SIAM Journal on Optimization, 15(3), pp. 720-750, 2005.

[8] Outrata, J., Kocvara, M. \& Zowe, J., Nonsmooth Approach to Optimization Problems with Equilibrium Constraints: Theory, Applications and Numerical Results. Kluwer Academic Publishers: Dordrecht, 1998.

[9] Raghunathan, A.U. \& Biegler, L.T., MPEC Formulations and Algorithms in Process Engineering. Computers and Chemical Engineering, 27, pp. 13811392, 2003.

[10] Raghunathan, A.U., Perez-Correa, J.R. \& Biegler, L.T., Data Reconciliation and Parameter Estimation in Flux-Balance Analysis. Biotechnology and Bioengineering, 84, pp. 700-709, 2003.

[11] Wächter, A. \& Biegler, L.T., On the Implementation of an Interior-Point Filter Line-Search Algorithm for Large-Scale Nonlinear Programming. Mathematical Programming, 106(1), pp. 25-57, 2006.

[12] Byrd, R., Hribar, M.E. \& Nocedal, J., An Interior Point Method for Large Scale Nonlinear Programming. SIAM Journal on Optimization, 9(4), pp. 877-900, 1999.

[13] DeMiguel, V., Friedlander, M.P., Nogales, F.J. \& Scholtes, S., A two-sided relaxation scheme for mathematical programs with equilibrium constraints. SIAM Journal on Optimization, 16(2), pp. 587-609, 2005. 